PROCEEDINGS OF THE

AMERICAN MATHEMATICAL SOCIETY

Volume 133, Number 12, Pages 3589-3596

S 0002-9939(05)07940-2

Article electronically published on June 28, 2005

\title{
INVARIANT SUBSPACES FOR A CLASS OF COMPLETE PICK KERNELS
}

\author{
MICHAEL T. JURY
}

(Communicated by Joseph A. Ball)

\begin{abstract}
Motivated by the work of McCullough and Trent, we investigate the $z$-invariant subspaces of the Hilbert function spaces associated to the Szego" kernels on the open unit disk. In particular, we characterize those kernels for which the the $z$-invariant subspaces are hyperinvariant, and (partially) those for which the so-called BLH subspaces are cyclic, obtaining counterexamples to two questions posed by McCullough and Trent.
\end{abstract}

\section{INTRODUCTION}

Fix a set $\Omega$ and a point $\omega \in \Omega$. Let $k(y, x)$ be a positive definite kernel on $\Omega$, normalized so that $k(\cdot, \omega) \equiv 1$. We say that $k$ is a complete Pick kernel if there exists a positive semidefinite function $b: \Omega \times \Omega \rightarrow \mathbb{C}$ with $|b(x, y)|<1$ such that

$$
1-\frac{1}{k(y, x)}=b(y, x)
$$

for all $x$ and $y$ in $\Omega$. Since $b(y, x)$ is positive semidefinite, there exists an index set $\mathcal{B}$ and functions $b_{j}: \Omega \rightarrow \mathbb{C}, j \in \mathcal{B}$, such that

$$
b(y, x)=\sum_{j \in \mathcal{B}} b_{j}(y) \overline{b_{j}(x)} .
$$

McCullough and Trent show in [10 that in this case each function $b_{j}$ defines a multiplier $M_{b_{j}}$ of the associated Hilbert function space $H(k)$, and they prove the following analogue of the Beurling-Lax-Halmos theorem:

Theorem 1. Let $k$ be a complete Pick kernel on $\Omega$, and for a Hilbert space $\mathcal{E}$ let $H_{\mathcal{E}}(k)$ denote the Hilbert space of $\mathcal{E}$-valued functions $H(k) \otimes \mathcal{E}$. Let $\mathcal{M}$ be a closed subspace of $H_{\mathcal{E}}(k)$. Then the following are equivalent:

(i) $\mathcal{M}$ is invariant for each $M_{b_{j}}$.

(ii) There exists an auxiliary Hilbert space $\mathcal{F}$ and an inner multiplier $\Phi: \Omega \rightarrow$ $\mathcal{L}(\mathcal{F}, \mathcal{E})$ such that

$$
\mathcal{M}=\Phi H_{\mathcal{F}}(k) .
$$

(iii) $\mathcal{M}$ is invariant for every multiplier $M_{\phi}$ of $H(k)$.

Received by the editors July 14, 2000 and, in revised form, July 16, 2004.

2000 Mathematics Subject Classification. Primary 47B32; Secondary 47A15, 47A16.

Key words and phrases. Pick kernel, invariant space, cyclic vector.

(C)2005 American Mathematical Society 
Now consider $\Omega=\mathbb{D}$ (the open unit disk) and let $s$ be the Szegő kernel

$$
s(z, w)=\frac{1}{1-z \bar{w}} .
$$

The kernel $s$ is a complete Pick kernel, and the space $H(s)$ is the usual Hardy space $H^{2}$, the space of analytic functions on $\mathbb{D}$ with square-summable power series. In this case it is possible to show that one may always choose $\mathcal{F}$ to be a subspace of $\mathcal{E}$, recovering the usual Beurling-Lax-Halmos theorem. Additionally, in the Hardy space the multiplier $\Phi(\zeta)$ is a coisometry almost everywhere on the unit circle. W. Arveson proved a special case of Theorem 1 in [4, and showed that in quite general circumstances $\operatorname{dim} \mathcal{F}$ must be infinite even when $\operatorname{dim} \mathcal{E}=1$; nonetheless if $\operatorname{dim} \mathcal{E}$ is finite and $\mathcal{M}$ has finite codimension, we can choose $\mathcal{F}$ to be finite dimensional. See [4] for details. Greene, Richter, and Sundberg [7] have shown that when $\Omega=\mathbb{B}^{d}$ (the unit ball of $\mathbb{C}^{d}$ ) and the kernel $k$ satisfies some mild additional assumptions, the vector-valued multiplier $\Phi(\zeta)$ is a coisometry almost everywhere on the boundary of $\mathbb{B}^{d}$, strengthening the analogy with the usual Beurling theorem.

In the case of the Szego" kernel, the function $b$ is $b(z, w)=z \bar{w}$. Thus the theorem says in particular that every $M_{z}$-invariant subspace of $H^{2}$ is hyperinvariant (i.e. invariant for every bounded operator on $H^{2}$ that commutes with $M_{z}$-here, the multipliers are $\left.M_{\phi}, \phi \in H^{\infty}\right)$. McCullough and Trent ask in [10] if every $M_{z^{-}}$ invariant subspace of $H(k)$ is hyperinvariant whenever $k$ is a complete analytic Pick kernel on $\mathbb{D}$ and multiplication by $z$ is bounded on $H(k)$. We show that this is not true in general, and give necessary and sufficient conditions for this to hold when the function $b$ has the form

$$
b(z, w)=f(z) \overline{f(w)}
$$

for a univalent analytic function $f: \mathbb{D} \rightarrow \mathbb{D}$.

$\mathrm{M}^{\mathrm{c}}$ Cullough and Trent also investigate the cyclicity of the subspaces described by Theorem 1 (hereafter called BLH subspaces). They show that if $k$ is a complete Pick kernel on $\mathbb{D}, M_{z}$ is bounded above and below, and $\mathcal{M}$ is a BLH subspace, then the space $\mathcal{M} \ominus z \mathcal{M}$ is one dimensional (i.e. $M_{z}$ has the codimension one property). When $k$ is the Szego" kernel, a nonzero vector in $\mathcal{M} \ominus z \mathcal{M}$ is cyclic for $M_{z}$ (the unilateral shift) restricted to $\mathcal{M}$. They ask if $M_{z}$ is cyclic on the BLH subspaces for more general $k$, at least when $k$ is a total Pick kernel (defined later) and $M_{z}$ is bounded above and below. Again, by appropriate choice of the function $f$, we provide a counterexample. However, a complete description of the kernels for which $M_{z}$ is cyclic on the BLH subspaces, even for this special case, seems elusive.

Let $\Omega$ be a set. We say a function $k: \Omega \times \Omega \rightarrow \mathbb{C}$ is a positive definite kernel on $\Omega$ if for each finite set $\left\{x_{1}, \ldots, x_{n}\right\} \subseteq \Omega$, the matrix

$$
\left(k\left(x_{i}, x_{j}\right)\right)_{i, j=1}^{n}
$$

is positive definite. For each $x \in \Omega$, define a function $k(\cdot, x)$ on $\Omega$ by $k(\cdot, x)(y)=$ $k(y, x)$. Define an inner product on the linear span of these functions by

$$
\left\langle\sum_{i} a_{i} k\left(\cdot, x_{i}\right), \sum_{j} b_{j} k\left(\cdot, x_{j}\right)\right\rangle=\sum_{i, j} a_{i} \overline{b_{j}} k\left(x_{j}, x_{i}\right) .
$$

Let $H(k)$ denote the Hilbert space obtained by completing the linear span of the functions $k(\cdot, x)$ with respect to the inner product $\langle\cdot, \cdot\rangle$. We may regard vectors $f$ in $H(k)$ as functions on $\Omega$, with $f(x)=\langle f, k(\cdot, x)\rangle$. 
A function $\phi$ in $H(k)$ is called a multiplier of $H(k)$ if $\phi g \in H(k)$ for every $g \in H(k)$. We then define the operator $M_{\phi}: H(k) \rightarrow H(k)$ by $M_{\phi} g=\phi g, g \in H(k)$; boundedness of $M_{\phi}$ follows from the closed graph theorem. Mult $(H(k))$ will denote the algebra of multipliers $\left\{M_{\phi}: \phi\right.$ is a multiplier of $\left.H(k)\right\}$. The Pick problem is to determine, given points $x_{1}, \ldots, x_{n}$ in $\Omega$ and complex numbers $\lambda_{1}, \ldots, \lambda_{n}$, if there exists a multiplier $\phi$ on $H(k)$ with $\left\|M_{\phi}\right\| \leq 1$ such that $\phi\left(x_{i}\right)=\lambda_{i}$ for each $i$. We may also formulate a matrix-valued version of this problem: for a positive integer $m$, a multiplier on the Hilbert space $H(k) \otimes \mathbb{C}^{m}$ is an $m \times m$ matrix-valued function $\Phi$ on $\Omega$ such that

$$
\Phi\left(\begin{array}{c}
f_{1} \\
\vdots \\
f_{m}
\end{array}\right) \in H(k) \otimes \mathbb{C}^{m} .
$$

As in the scalar case, we define the operator $M_{\Phi}$ of "multiplication by $\Phi$ ". Now, given points $x_{1}, \ldots, x_{n}$ and $m \times m$ matrices $\Lambda_{1}, \ldots, \Lambda_{n}$, we ask whether or not there exists a multiplier $\Phi$ on $H(k) \otimes \mathbb{C}^{m}$ with $\left\|M_{\Phi}\right\| \leq 1$ such that $\Phi\left(x_{i}\right)=\Lambda_{i}$.

Definition 1. A kernel $k$ on $\Omega$ has the $m \times m$ Pick property if, for any finite set of points $x_{1}, \ldots, x_{n}$ in $\Omega$ and any choice of $m \times m$ matrices $\Lambda_{1}, \ldots, \Lambda_{n}$, the following are equivalent:

(1) There exists a multiplier $\Phi$ on $H(k) \otimes \mathbb{C}^{m}$ such that $\left\|M_{\Phi}\right\| \leq 1$ and $\Phi\left(x_{i}\right)=$ $\Lambda_{i}$ for each $i$.

(2) The $m n \times m n$ matrix $\left(1-\Lambda_{j}^{*} \Lambda_{i}\right) k\left(x_{j}, x_{i}\right)$ is positive semidefinite.

A kernel $k$ has the complete Pick property if it has the $m \times m$ Pick property for every $m$.

Fix a set $\Omega$ and a point $\omega \in \Omega$. As before we assume $k$ is normalized so that $k(\cdot, \omega) \equiv 1 . k$ has the complete Pick property if and only if there exists a positive semidefinite function $b: \Omega \times \Omega \rightarrow \mathbb{C}$ with $|b(x, y)|<1$ such that

$$
1-\frac{1}{k(y, x)}=b(y, x)
$$

for all $x$ and $y$ in $\Omega$. (See 11, 12, 9]; see also 2.) Since $b(y, x)$ is positive semidefinite, there is an index set $\mathcal{B}$ and functions $b_{j}: \Omega \rightarrow \mathbb{C}, j \in \mathcal{B}$, such that

$$
b(y, x)=\sum b_{j}(y) \overline{b_{j}(x)} .
$$

Following [10, a complete Pick kernel $k$ is called total if whenever $\mathcal{M}$ and $\mathcal{N}$ are BLH subspaces with $\mathcal{M} \subseteq \mathcal{N} \subseteq H(k)$, the kernel

$$
\frac{P_{\mathcal{M}} k(z, w)}{P_{\mathcal{N}} k(z, w)}
$$

is positive semidefinite. (Here $P_{\mathcal{M}}$ denotes orthogonal projection onto $\mathcal{M}$.) For example, the Szegö kernel is a total Pick kernel, as is shown in the next section using Beurling's theorem and the divisibility properties of inner functions.

\section{MAIN RESUlts}

From now on we restrict ourselves to the following situation: $f$ will be a univalent analytic function from $\mathbb{D}$ into itself, and we will assume for convenience that $f(0)=$ 
0 . Let $G$ denote the image of $\mathbb{D}$ under the conformal mapping $f$. Define a positive definite kernel $k_{f}: \mathbb{D} \times \mathbb{D} \rightarrow \mathbb{C}$ by

$$
k_{f}(z, w)=\frac{1}{1-f(z) \overline{f(w)}} .
$$

Choosing the base point $\omega=0$ (so that $k_{f}(\cdot, 0) \equiv 1$ ), (11) is easily seen to hold with $b(z, w)=f(z) \overline{f(w)}$. The kernels $k_{f}$ are called Szegö kernels, because they may be regarded as restrictions of the Szegö kernel $s$ to the subset $G=f(\mathbb{D}) \subseteq \mathbb{D}$ (see [2]).

In this situation, we show that $M_{z}$ is a multiplier of $H\left(k_{f}\right)$ if and only if there exists a function $\psi \in H^{\infty}(\mathbb{D})$ such that $\left.\psi\right|_{G}=f^{-1}$, and that every $M_{z}$-invariant subspace of $H\left(k_{f}\right)$ is hyperinvariant (i.e. left invariant by every bounded operator on $H\left(k_{f}\right)$ that commutes with $\left.M_{z}\right)$ if and only if this function $\psi$ is a weak-star generator of $H^{\infty}(\mathbb{D})$. (A function $\phi$ is called a weak-star generator of $H^{\infty}(\mathbb{D})$ if the polynomials in $\phi$ are weak-star dense in $H^{\infty}(\mathbb{D})$.) Furthermore, we show that $M_{z}$ is cyclic when restricted to the BLH subspaces if and only if the analytic Toeplitz operator $T_{\psi}$ is cyclic on $H^{2}$.

To prove these results, we make use of the following propositions which describe the multipliers of $H\left(k_{f}\right)$ :

Proposition 1. A bounded analytic function $\phi$ on $\mathbb{D}$ is a multiplier of $H\left(k_{f}\right)$ if and only if there exists a function $\psi \in H^{\infty}$ such that $\phi \circ f^{-1}=\left.\psi\right|_{G}$.

Proof. We exploit the fact that the kernel $k_{f}$ and the Szegö kernel $s$ have the Pick property. Suppose $\phi$ is a multiplier of $H\left(k_{f}\right)$ with $\left\|M_{\phi}\right\|=M$, and let $z_{1}, \ldots, z_{n}$ be points in $\mathbb{D}$. By computing the Gramian of $M^{2} \cdot I-M_{\phi} M_{\phi^{*}}$ with respect to the set of vectors $\left\{k_{f}\left(\cdot, z_{j}\right)\right\}$, we see that the matrix $\left(A_{i j}\right)_{i, j=1}^{n}$ with $(i, j)$ entry given by

$$
A_{i j}=\left(M^{2}-\phi\left(z_{i}\right) \overline{\phi\left(z_{j}\right)}\right) k_{f}\left(z_{i}, z_{j}\right)
$$

is positive semidefinite. Let $\zeta_{i}=f\left(z_{i}\right)$. Then

$$
\begin{aligned}
A_{i j} & =\left(M^{2}-\left(\left(\phi \circ f^{-1}\right)\left(\zeta_{i}\right) \overline{\left(\phi \circ f^{-1}\right)\left(\zeta_{j}\right)}\right)\right) k_{f}\left(f^{-1}\left(\zeta_{i}\right), f^{-1}\left(\zeta_{j}\right)\right) \\
& =\left(M^{2}-\left(\left(\phi \circ f^{-1}\right)\left(\zeta_{i}\right) \overline{\left(\phi \circ f^{-1}\right)\left(\zeta_{j}\right)}\right)\right) \frac{1}{\left(1-\zeta_{i} \overline{\zeta_{j}}\right)} \\
& =\left(M^{2}-\left(\left(\phi \circ f^{-1}\right)\left(\zeta_{i}\right) \overline{\left(\phi \circ f^{-1}\right)\left(\zeta_{j}\right)}\right)\right) s\left(\zeta_{i}, \zeta_{j}\right) .
\end{aligned}
$$

Thus, by the Pick property for $s$, there exists a function $h \in H^{\infty},\|h\|_{\infty} \leq M$, such that $h\left(\zeta_{i}\right)=\left(\phi \circ f^{-1}\right)\left(\zeta_{i}\right)$. Now let $\Lambda=\left\{\lambda_{i}: i \in \mathbb{N}\right\}$ be a set of uniqueness for analytic functions on $G$, and let $h_{N}$ be the function obtained in the manner above with $\zeta_{i}=\lambda_{i}, i=1, \ldots, N$. Since $\left\|h_{N}\right\|_{\infty} \leq M$ for each $N$, by the BanachAlaoglu theorem there exists a subsequence $\left(h_{N_{j}}\right)_{j=1}^{\infty}$ of $\left(h_{N}\right)_{N=1}^{\infty}$ which is weak-star convergent in $H^{\infty}$ to a function $\psi$. We have $\|\psi\|_{\infty} \leq M$, and $\psi\left(\lambda_{i}\right)=\left(\phi \circ f^{-1}\right)\left(\lambda_{i}\right)$ for each $\lambda_{i} \in \Lambda$. Since $\Lambda$ is a set of uniqueness for $G,\left.\psi\right|_{G}=\phi \circ f^{-1}$. The reverse implication is proved by similar reasoning, reversing the roles of $k_{f}$ and $s$ and using the fact that $k_{f}$ is a Pick kernel.

The preceding argument is standard in spaces possessing complete Pick kernels; a more general form of this proposition can be found in 5 .

For $\psi \in H^{\infty}(\mathbb{D})$, the operator $T_{\psi}: H^{2} \rightarrow H^{2}$ defined by $T_{\psi} f=\psi f$ is called the analytic Toeplitz operator with symbol $\psi$. The map $\psi \rightarrow T_{\psi}$ is an isometric 
Banach algebra isomorphism and a weak-star homeomorphism (see Hoffman [8]). We now show that each multiplier $M_{\phi}$ of $H\left(k_{f}\right)$ is unitarily equivalent to an analytic Toeplitz operator.

Proposition 2. Let $\phi$ be a multiplier of $H\left(k_{f}\right)$ and let $\psi$ be the analytic continuation of $\phi \circ f^{-1}$ to $\mathbb{D}$, as in Proposition 1 . Then $M_{\phi} \cong T_{\psi}$.

Proof. The linear span of the functions $\{s(\cdot, \lambda): \lambda \in G\}$ is dense in $H^{2}$, for if $f(\lambda)=\langle f, s(\cdot, \lambda)\rangle_{H^{2}}=0$ for all $\lambda$ in $G$, then $f \equiv 0$ on $G$ and hence on $\mathbb{D}$, since $G$ is open.

Define a map $V$ from $\bigvee\{s(\cdot, \lambda): \lambda \in G\}$ to $\bigvee\left\{k_{f}(\cdot, w): w \in \mathbb{D}\right\}$ by setting $V s(\cdot, \lambda)=k_{f}\left(\cdot, f^{-1}(\lambda)\right)$ and extending linearly. Since

$$
\begin{aligned}
\langle V s(\cdot, \lambda), V s(\cdot, \mu)\rangle_{H\left(k_{f}\right)} & =\left\langle k_{f}\left(\cdot, f^{-1}(\lambda)\right), k_{f}\left(\cdot, f^{-1}(\mu)\right)\right\rangle \\
& =k_{f}\left(f^{-1}(\mu), f^{-1}(\lambda)\right) \\
& =\frac{1}{1-\mu \bar{\lambda}} \\
& =\langle s(\cdot, \lambda), s(\cdot, \mu)\rangle_{H^{2}},
\end{aligned}
$$

$V$ is an isometry from a dense subset of $H^{2}$ onto a dense subset of $H\left(k_{f}\right)$, so it extends to a unitary operator (which we also denote by $V$ ) from $H^{2}$ onto $H\left(k_{f}\right)$. It is now easy to verify that $T_{\psi}=V^{*} M_{\phi} V$.

The proof shows that every function in $H\left(k_{f}\right)$ has the form $g \circ f$, with $g \in H^{2}$, and $\|g \circ f\|_{H\left(k_{f}\right)}=\|g\|_{H^{2}}$ (this is evident for kernel functions and follows in general by taking limits). the proposition also shows that every analytic Toeplitz operator gives rise to a multiplier of $H\left(k_{f}\right)$ :

Corollary 1. Let $V$ be as in the proposition. For any $\psi \in H^{\infty}, \psi \circ f$ is a multiplier of $H\left(k_{f}\right)$ and $V T_{\psi} V^{*}=M_{\psi \circ f}$.

Proof. This is an immediate consequence of the preceding remark; alternatively we could observe that $(\psi \circ f) \circ f^{-1}=\left.\psi\right|_{G}$ and apply Propositions 1 and 2 ,

From now on we assume that $f$ is such that $M_{z}$ is bounded on $H\left(k_{f}\right)$; by Proposition 1 this means that there exists an $H^{\infty}$ function $\psi$ on $\mathbb{D}$ such that $\left.\psi\right|_{G}=f^{-1}$. The next proposition deals with the set of bounded operators that commute with $M_{z}$ (here $\{T\}^{\prime}$ denotes the set of bounded operators that commute with the operator $T)$ :

Proposition 3. Suppose $M_{z}$ is bounded on $H\left(k_{f}\right)$ with $M_{z} \cong T_{\psi}$. If $\psi$ is univalent as an analytic function on $\mathbb{D}$, then $\left\{M_{z}\right\}^{\prime}=\operatorname{Mult}\left(H\left(k_{f}\right)\right)$.

Proof. Since every multiplier commutes with $M_{z}$, it is enough to show that $\left\{M_{z}\right\}^{\prime} \subseteq$ $\operatorname{Mult}\left(H\left(k_{f}\right)\right)$; by Proposition 2 and its corollary, this is equivalent to $\left\{T_{\psi}\right\}^{\prime} \subseteq$ $\operatorname{Mult}\left(H^{2}\right)$. So suppose $A$ is a bounded operator on $H^{2}$ that commutes with $T_{\psi}$. Then $A^{*}$ commutes with $T_{\psi}^{*}$, and hence with $T_{\psi}^{*}-\overline{\psi(\lambda)} I$ for every $\lambda \in \mathbb{D}$. Thus $\operatorname{ker}\left(T_{\psi}^{*}-\overline{\psi(\lambda)} I\right)$ is invariant for $A^{*}$. Since $\psi$ was assumed univalent, $\operatorname{ker}\left(T_{\psi}^{*}-\overline{\psi(\lambda)} I\right)$ is the one-dimensional space spanned by $s(\cdot, \lambda)$. Thus $A^{*} s(\cdot, \lambda)=\overline{a(\lambda)} s(\cdot, \lambda)$ for some complex number $\overline{a(\lambda)}$. Now for every $g \in H^{2}$ and every $\lambda \in \mathbb{D}$, we have

$$
\langle A g, s(\cdot, \lambda)\rangle=\left\langle g, A^{*} s(\cdot, \lambda)\right\rangle=a(\lambda) g(\lambda) .
$$

So $a g=A g$ is in $H^{2}$ and $A=T_{a}$. 
The fact that $\left\{T_{\psi}\right\}^{\prime}=\operatorname{Mult}\left(H^{2}\right)$ when $\psi$ is univalent is actually a special case of a much more general theorem about the commutant of an analytic Toeplitz operator; see [17. The proof also shows that in this situation, $M_{z}^{*}$ is in the CowenDouglas class (see [6]).

Our first result will invoke the following theorem of D. Sarason [13]:

Theorem 2. Let $\psi \in H^{\infty}$. Then $T_{\psi}$ has the same invariant subspaces as $T_{z}$ if and only if $\psi$ is a weak-star generator of $H^{\infty}$.

We can now describe those kernels $k_{f}$ for which every $M_{z}$-invariant subspace is hyperinvariant:

Theorem 3. With notations as above, the following are equivalent:

(1) Every $M_{z}$-invariant subspace of $H\left(k_{f}\right)$ is hyperinvariant.

(2) Every $M_{z}$-invariant subspace of $H\left(k_{f}\right)$ is $M_{f}$-invariant.

(3) Every $T_{\psi}$-invariant subspace of $H^{2}$ is $T_{z}$-invariant.

(4) $\psi$ is a weak-star generator of $H^{\infty}$.

Proof. (1) $\Rightarrow(2)$ is trivial. By Proposition 2, $M_{z} \cong T_{\psi}$ and $M_{f} \cong T_{z}$; the equivalence of $(2)$ and (3) follows. (3) $\Leftrightarrow(4)$ is just an application of Theorem 2 above, together with the fact that $T_{z}$-invariant subspaces are invariant for every analytic Toeplitz operator, the consequence of Beurling's theorem discussed earlier.

It remains to show $(2) \Rightarrow(1)$. Since $(2) \Leftrightarrow(4)$, the function $\psi$ is a weak-star generator of $H^{\infty}$ and hence is univalent in $\mathbb{D}$ (see [14), so by Proposition 3] the only bounded operators on $H\left(k_{f}\right)$ that commute with $M_{z}$ are the multipliers. By Theorem 1] each $M_{f}$-invariant subspace is invariant for every multiplier, and (1) follows.

Turning now to cyclic vectors, we show that the kernels $k_{f}$ are total Pick kernels, and describe the cyclicity of $M_{z}$ on BLH subspaces in terms of the cyclicity of analytic Toeplitz operators.

Proposition 4. The kernels $k_{f}$ are total Pick kernels.

Proof. We observe that $\mathcal{M}$ is a BLH subspace of $H\left(k_{f}\right)$ if and only if $V^{*} \mathcal{M}$ is a BLH subspace (i.e. shift invariant subspace) of $H^{2}$, where $V$ is the unitary map of Proposition 2. By Beurling's theorem, $V^{*} \mathcal{M}=\phi H^{2}$, where $\phi$ is an inner function, so

$$
\mathcal{M}=V V^{*} \mathcal{M}=V \phi H^{2}=(\phi \circ f) H\left(k_{f}\right) .
$$

A straightforward calculation then shows that

$$
P_{\mathcal{M}} k_{f}(z, w)=\phi(f(z)) \overline{\phi(f(w))} k_{f}(z, w) .
$$

Recall that if $\phi_{1}$ and $\phi_{2}$ are inner functions and $\phi_{1} H^{2} \subseteq \phi_{2} H^{2}$, then $\phi_{1} / \phi_{2}$ is inner. Let $\mathcal{M}$ and $\mathcal{N}$ be BLH subspaces of $H\left(k_{f}\right)$ with $\mathcal{M} \subseteq \mathcal{N}$, so there exist inner functions $\phi_{1}$ and $\phi_{2}$ with $\phi_{2}$ dividing $\phi_{1}$ so that $\mathcal{M}=\left(\phi_{1} \circ f\right) H\left(k_{f}\right)$ and $\mathcal{N}=\left(\phi_{2} \circ f\right) H\left(k_{f}\right)$. Then

$$
\begin{aligned}
\frac{P_{\mathcal{M}} k_{f}(z, w)}{P_{\mathcal{N}} k_{f}(z, w)} & =\frac{\phi_{1}(f(z)) \overline{\phi_{1}(f(w))} k_{f}(z, w)}{\phi_{2}(f(z)) \overline{\phi_{2}(f(w))} k_{f}(z, w)} \\
& =\frac{\phi_{1}}{\phi_{2}}(f(z)) \overline{\frac{\phi_{1}}{\phi_{2}}(f(w))}
\end{aligned}
$$


The last expression is a positive semidefinite function on $\mathbb{D} \times \mathbb{D}$, so $k_{f}$ is a total Pick kernel.

Theorem 4. Let $k_{f}$ be a complete Pick kernel, and suppose $M_{z}$ is bounded, so that $M_{z} \cong T_{\psi}$. Then the following are equivalent:

(1) $M_{z}$ is cyclic on some $B L H$ subspace $\mathcal{M} \subseteq H\left(k_{f}\right)$.

(2) $M_{z}$ is cyclic on every $B L H$ subspace $\mathcal{M} \subseteq H\left(k_{f}\right)$.

(3) $T_{\psi}$ is cyclic on $H^{2}$.

Proof. In the proof of Proposition 4 we showed that the BLH subspaces of $H\left(k_{f}\right)$ have the form $(\phi \circ f) H\left(k_{f}\right)$, where $\phi$ is an inner function. From this it follows that a function $g$ in a BLH subspace $\mathcal{M} \subseteq H\left(k_{f}\right)$ is cyclic for $\left.M_{z}\right|_{\mathcal{M}}$ if and only if $g /(\phi \circ f)$ is cyclic for $M_{z}$ on $H\left(k_{f}\right)$, and from this follows the equivalence of assertions (1) and (2). Since $M_{z} \cong T_{\psi}, M_{z}$ is cyclic on the BLH subspaces if and only if $T_{\psi}$ is cyclic on $H^{2}$.

\section{EXAMPLES AND REMARKS}

Using Theorem 3 we can construct an analytic Pick kernel for which $M_{z}$ is bounded but for which there exist $M_{z}$-invariant subspaces which are not hyperinvariant. To do this, we need only exhibit a univalent analytic function $f: \mathbb{D} \rightarrow G$, (with $G \subseteq \mathbb{D}$ ) so that $f^{-1}$ extends to a function $\psi \in H^{\infty}$ which is not a weak-star generator of $H^{\infty}$.

Consider a bounded, simply-connected domain $\Omega \subseteq \mathbb{C}$, and let $\psi$ be a Riemann map from $\mathbb{D}$ onto $\Omega$. Sarason shows in 14 . that $\psi$ fails to be a weak-star generator of $H^{\infty}$ if and only if $\Omega$ has the following property:

(*) There exists a domain $B$ containing $\Omega$ properly such that $\sup _{z \in B}|f(z)|=$ $\sup _{z \in \Omega}|f(z)|$ for all $f$ bounded and analytic in $B$.

For example, let $B$ be an open disk, and fix a point $z_{0} \in B$ (not the center). Let $\alpha=\operatorname{dist}\left(z_{0}, \partial B\right)$ and let $\bar{D}$ be the closed disk of radius $\alpha$ centered at $z_{0}$. Then $B \backslash \bar{D}$ has property (*), by the maximum modulus principle.

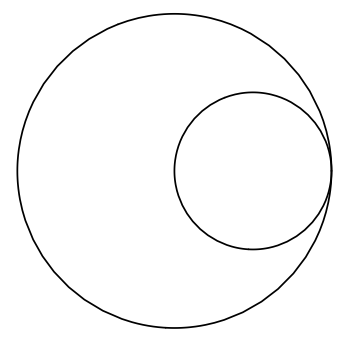

Figure 1. The domain $B \backslash \bar{D}$

We now let $\Omega$ be a domain satisfying $(*)$ with $\mathbb{D} \subseteq \Omega$ and let $\psi: \mathbb{D} \rightarrow \Omega$ be a Riemann map. Taking $f$ to be the restriction of $\psi^{-1}$ to $\mathbb{D}$ does the job.

Regarding cyclic vectors, we can use Theorem 4 to exhibit a total Pick kernel for which multiplication by $z$ is bounded above and below, but such that $M_{z}$ is not cyclic on any of the BLH subspaces. By Theorem 4 it will suffice to exhibit an analytic Toeplitz operator which is bounded below but is not cyclic. For this a Riemann map $\phi$ of $\mathbb{D}$ onto a slit disk which contains $\mathbb{D}$ suffices (see [16]); we then let 
$f=\left.\phi^{-1}\right|_{\mathbb{D}}$ as in the previous example. The cyclicity of analytic Toeplitz operators is a very subtle problem, which has not been solved completely; it is for this reason that we regard the "characterization" of Theorem 4 as incomplete. We remark, however, that $T_{\psi}$ is cyclic when $\psi$ is a weak-star generator of $H^{\infty}$, so combining Theorem 3 and the above remarks tells us that if every $M_{z}$-invariant subspace of $H\left(k_{f}\right)$ is hyperinvariant (and hence a BLH subspace), then $M_{z}$ is cyclic on each of these spaces.

In general, we expect the kernels $k_{f}$ to be a good source of counterexamples for questions regarding multiplication by $z$ on spaces possessing a complete Pick kernel: as seen in these two examples, one can choose $f$ so that $M_{z}$ is unitarily equivalent to an analytic Toeplitz operator with "bad" properties.

\section{REFERENCES}

[1] Agler, J. and McCarthy, J. E.. Pick interpolation and Hilbert function spaces, American Mathematical Society, Providence, RI, 2002. MR1882259 (2003b:47001)

[2] Agler, J. and McCarthy, J. E., Complete Nevanlinna-Pick kernels, Journal of Functional Analysis 175 (2000), 111-124. MR1774853 (2001h:47019)

[3] Aleman, A., Richter, S., and Sundberg, C., The majorization function and the index of invariant subspaces of the Bergman space, Journal Analyse de Mathématique 86 (2002), 139-182. MR.1894480 (2003g:30058)

[4] Arveson, W., The curvature invariant of a Hilbert module over $\mathbb{C}\left[z_{1}, \cdots, z_{d}\right]$, J. Reine. Angew. Math. 522 (2000), 173-236. MR1758582 (2003a:47013)

[5] Ball, J., Trent, T., and Vinnikov, V., Interpolation and commutant lifting for multipliers on reproducing kernel Hilbert spaces, in Operator Theory and Analysis (Amsterdam 1997), OT122, 89-138, Birkhäuser, 2001. MR1846055 (2002f:47028)

[6] Cowen, M. J. and Douglas, R. G., Complex geometry and operator theory, Acta Mathematica 141 (1978), 187-261. MR.0501368 (80f:47012)

[7] Greene, D., Richter, S., and Sundberg, C., The structure of inner multipliers on spaces with complete Nevanlinna-Pick kernels, Journal of Functional Analysis 194 (2002), 311-331. MR.1934606 (2003h:46038)

[8] Hoffman, K., Banach Spaces of Analytic Functions, Prentice-Hall, Englewood Cliffs, NJ, 1962. MR0133008 (24:A2844)

[9] McCullough, S., The local deBranges-Rovnyak construction and complete Nevanlinna-Pick kernels, in Algebraic Methods in Operator Theory, 15-24, Birkhäuser, 1994. MR 1284929 (95j:47016)

[10] McCullough, S., and Trent, T., Invariant subspaces and Nevanlinna-Pick kernels, Journal of Functional Analysis 178 (2000), 226-249. MR1800795 (2002b:47006)

[11] Quiggin, P., For which reproducing kernel Hilbert spaces is Pick's theorem true?, Integral Equations and Operator Theory 16 (1993), 244-266. MR1205001 (94a:47026)

[12] Generalisations of Pick's theorem to reproducing kernel Hilbert spaces, Ph.D. thesis, Lancaster University, 1994.

[13] Sarason, D., Invariant subspaces and unstarred operator algebras, Pacific Journal of Mathematics 17 (1966), 511-517. MR0192365 (33:590)

[14] — Weak-star generators of $H^{\infty}$, Pacific Journal of Mathematics 17 (1966), 519-528. MR0211269 (35:2151)

[15] Generalized interpolation in $H^{\infty}$, American Mathematical Society Transactions 27 (1967), 180-203. MR0208383 (34:8193)

[16] Solomyak, B. M. and Volberg, A. L., Multiplicity of analytic Toeplitz operators, in Toeplitz operators and spectral function theory, 87-192, Birkhäuser, 1989. MR.1030051 (91g:47021)

[17] Thomson, J. E., The commutant of a class of analytic Toeplitz operators II, Indiana University Mathematics Journal 25 (1976), 793-800. MR0417843 (54:5891)

Department of Mathematics, Purdue University, 150 N. University St., West LafayETTE, INDIANA 47907-2067

E-mail address: jury@math.purdue.edu 